\title{
Desenvolvimento e validação de um método analítico para quantificação de atrazina do fipronil e seus produtos de degradação em amostras de solo por cromatografia líquida de alta eficiência
}

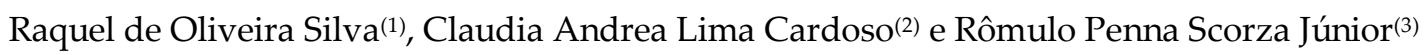 \\ 1,2Universidade Estadual de Mato Grosso do Sul. ${ }^{3}$ Embrapa Agropecuária Oeste, MS, Brasil
}

\begin{abstract}
Resumo
O uso de agrotóxicos na agricultura pode ser uma fonte de risco para a saúde humana e para o ambiente. Considerando que o solo é um dos principais destinos desses compostos após a aplicação, este estudo apresenta a validação de um método analítico para a quantificação dos resíduos de atrazina, fipronil e seus produtos de degradação (desulfinil, sulfeto e sulfona) em solo, empregando Cromatografia Líquida de Alta Eficiência (CLAE) com detector de arranjo de diodos (DAD). O método validado consiste na agitação por $1 \mathrm{~h}$ do solo + metanol, centrifugação por $30 \mathrm{~min}$, seguido de filtração. O método apresentou recuperações médias para o fipronil, dessulfinil, sulfeto, sulfona e atrazina entre 72 a 110\% para ambos os solos, com valores de coeficiente de variação inferiores a $13 \%$. Todas as curvas analíticas apresentaram coeficientes de determinação $\left(R^{2}\right)$ superiores a 0,999. Os limites de quantificação para ofipronil, dessulfinil, sulfeto e sulfona foram de $4 \mu g g^{-1}$ e para a atrazina de $2 \mu \mathrm{g} \mathrm{g}^{-1}$. O método validado mostrou-se adequado para ser aplicado com sucesso em amostras de solos.
\end{abstract}

Palavras-chave: agrotóxico, CLAE e solo.

\section{Abstract}

The agricultural use of pesticides can be a source of risks for human health and for the environment. Considering that soil is one of the main final destination of pesticides after application, this study presents the validation of an analytical method for atrazine, fipronil and its metabolites (desulfinyl, sulfide and sulfone) residues quantification in soil using High Performance Liquid Chromatography (HPLC) with diode array detector (DAD). The validated method consists in shaking soil + methanol for one hour, following extract centrifugation for 30 minutes and filtration. The method showed average recoveries between 79 and $110 \%$ for both soils and all pesticides, with variation coefficients below 13\%. All analytical calibration curves showed determination coefficients $\left(R^{2}\right)$ higher than 0.999. The quantification limit for fipronil, desulfinyl, sulfide and sulfone was $4 \mu \mathrm{g} \mathrm{g}^{-1}$ and for atrazine $2 \mu \mathrm{g} \mathrm{g}^{-1}$. The validated method showed adequate to be used with success in soil samples.

Keywords: Pesticide, HPLC, soil. 


\section{Introdução}

$\mathrm{O}$ uso de agrotóxicos na agricultura tem sido apontado como fonte de risco para a saúde humana e para o ambiente. Para avaliar e confirmar estes riscos é necessário o desenvolvimento de métodos analíticos confiáveis, com objetivo de identificação e quantificação dos agrotóxicos em diferentes matrizes, permitindo seu monitoramento. Análises de agrotóxicos apresentam elevado custo e consomem muito tempo (SPADOTTO et al., 2004). Considerando o grande número de agrotóxicos, bem como seus produtos de degradação, há necessidade do desenvolvimento de métodos confiáveis e rápidos.

O desenvolvimento e adaptação de métodos analíticos para identificação e quantificação de resíduos de agrotóxicos no solo apresentam dificuldades que envolvem as análises desses resíduos em matrizes sólidas, sua complexidade, tempo de extração e a quantidade utilizada de solventes (ANDREU \& PICÓ, 2004).

Estudos com o objetivo de validação de métodos analíticos para extração simultânea de compostos são importantes já que em um único sistema de produção agrícola, numa mesma safra, pode ocorrer a aplicação de vários agrotóxicos. Além disso, um método deve ser robusto para a extração desses resíduos em solos com diferentes teores de matéria orgânica $(\mathrm{MO})$, principal responsável pela sorção dos agrotóxicos não ionizáveis em solos. $\mathrm{O}$ maior teor de MO no solo pode promover aumento da sorção dos agrotóxicos e seus metabólitos, podendo influenciar na sua extração (PERES et al., 2002). Os agrotóxicos selecionados nesse estudo (fipronil e atrazina) são de grande importância e bastante utilizados na cultura da cana-de-açúcar em Mato Grosso do Sul.

O inseticida fipronil (5-amino-[2,6dicloro-4(trifluorometil) fenil]-4-trifluorometil-sulfinil-1Hpirazol-3carbonitrila) (Figura 1) têm sido comumente usado para o controle de insetospraga na cultura da cana-de-açúcar, sendo aplicado no solo e/ou na parte aérea das plantas (MAPA, 2015). É um inseticida que pertence a classe dos fenilpirazóis, possui baixa solubilidade em água $\left(2,4 \mathrm{mg} \mathrm{L}^{-1} \mathrm{em} \mathrm{pH} 5\right)$, coeficiente de sorção (Koc) igual a $803 \mathrm{~mL} \mathrm{~g}^{-1}$ e o $\log K_{\text {ow }}$ igual a 4,01. Assim, o fipronil apresenta um caráter hidrofóbico com baixo potencial de lixiviação e forte adsorção no solo e em sedimentos (BARCELÓ, 1997; BASF, 2015). O fipronil possui alta toxicidade para aves e moderada para mamíferos de pequeno porte. Durante a degradação podem formar-se quatro produtos, a saber: amida por hidrolise, dessulfinil por fotólise, sulfeto por redução e sulfona por oxidação (Figura 1) (BOBÉ et al., 1998). A importância de incluir os produtos de degradação do fipronil nesse estudo surge por eles serem igualmente ou mais tóxicos que o próprio fipronil (Pesticide Action Network-UK (PAN) 2000), por exemplo, o dessulfinil, que é 10 vezes mais tóxico a mamíferos que o fipronil (USEPA, 1996).

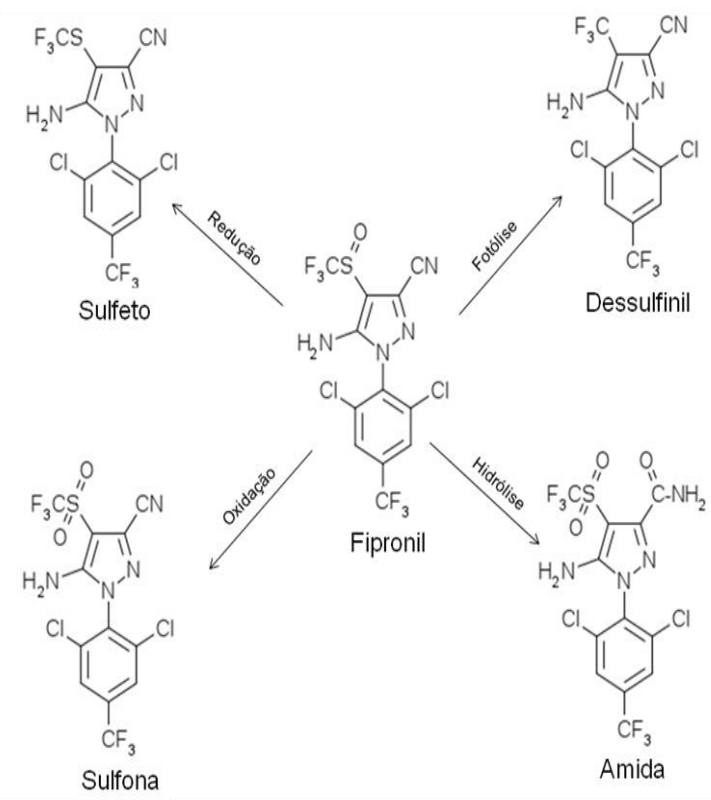

Figura 1: Estruturas químicas do fipronil e seus produtos de degradação amida, dessulfinil, sulfeto e sulfona.

A atrazina (2-cloro-4-etilamino-6isopropilamino-s-triazina) (Figura 2) pertence à família das triazinas e destaca-se como herbicida seletivo com aplicação recomendada em pré e pós-emergência para o controle de plantas daninhas nas culturas de cana-de-açúcar, milho, sorgo, pinus, seringueira e abacaxi (AGROFIT, 2003). A atrazina tem sido frequentemente 
detectada como o principal poluente orgânico de águas subterrâneas e superficiais em vários países (MARTINAZZO et al., 2011). É solúvel em água (solubilidade igual a $33 \mathrm{mg} \mathrm{L}^{-1}$ ), possui coeficiente de sorção (Koc) entre 0,4 e 4,5 $\mathrm{L} \mathrm{kg}^{-1} \mathrm{e}$ log Kow igual a 2,34 (MONTGOMERY, 1997) é um composto com alta mobilidade no solo e, portanto, alto potencial para contaminação de águas subterrâneas (ARIAS-ESTEVEZ et al., 2008; MARTINAZZO et al., 2011), além de ser considerado potencialmente cancerígeno e desregulador hormonal para aves, peixes e ratos (HAYES et al., 2011).

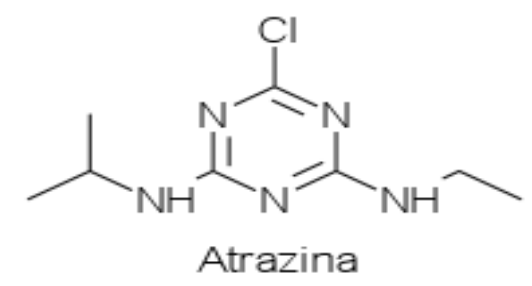

Figura 2. Estrutura química da atrazina.

O objetivo deste estudo foi desenvolver e validar um método para a quantificação simultânea por cromatografia líquida de alta eficiência (CLAE) de resíduos dos agrotóxicos atrazina, fipronil e seus produtos de degradação em dois tipos de solo utilizados para cultivo da cana-de-açúcar em Mato Grosso do Sul.

\section{Material e métodos}

Os padrões dos agrotóxicos utilizados foram doados pela BASF e adquiridos da Dr. Ehrenstorfer (Alemanha) com pureza superior a $97 \%$. As soluções-estoque dos agrotóxicos atrazina, fipronil e seus produtos de degradação (dessulfinil, sulfeto e sulfona) foram preparados na concentração de $1 \mathrm{mg} \mathrm{mL}^{-1} \mathrm{em}$ acetona grau HPLC e armazenadas a $-20^{\circ} \mathrm{C}$. Para identificação e quantificação dos agrotóxicos foi utilizado um CLAE, modelo Varian 920-LC, equipado com uma coluna de fase reversa C-18 Polaris $(25 \mathrm{~cm} \mathrm{x}$ $4,6 \mathrm{~mm} \times 5 \mu \mathrm{m})$, pré-coluna C-18 Polaris $(2,5 \mathrm{~cm}$ $x 4,6 \mathrm{~mm} \times 5 \mu \mathrm{m})$ e detector de arranjo de diodos (DAD).

No desenvolvimento da metodologia foram otimizadas as seguintes condições cromatográficas: fase móvel composta pela mistura metanol, água e acetonitrila (60:30:10, $\mathrm{v} / \mathrm{v})$, sendo metanol e acetonitrila grau HPLC (J.T. Baker, EUA), e água ultrapura obtida pelo sistema da Gehaka (Brasil) com resistividade de
$18 \mathrm{~m} \Omega \mathrm{cm}$, em modo de eluição isocrático $(1 \mathrm{~mL}$ $\left.\mathrm{min}^{-1}\right)$, temperatura do forno da coluna de $40^{\circ} \mathrm{C}$ com e volume de injeção de $20 \mu \mathrm{L}$ em pH 7 . Todos os agrotóxicos estudados absorvem em $220 \mathrm{~nm}$, comprimento de onda no qual foram determinados simultaneamente. A fase móvel, preparada diariamente, foi filtrada em membrana de $0,45 \mu \mathrm{m}$ (Millipore, USA) antes do uso e desgaseificada em ultrassom por 30 minutos. Nessas condições os tempos de retenção foram de 6,43; 11,81; 12,88; 14,55 e 16,55 mim para atrazina, dessulfenil, fipronil, sulfeto e sulfona, respectivamente. Esta metodologia analítica foi validada de acordo os parâmetros de seletividade, linearidade, limite de detecção, limite de quantificação, exatidão e precisão.

A seletividade foi avaliada empregando análises das amostras sem adição dos padrões (amostras testemunha). Para determinação da linearidade foram preparadas soluções padrão a partir das soluções-estoque por diluição em metanol grau HPLC nas concentrações de 1,0; 2,$0 ; 5,0 ; 10,0 ;$ e $20,0 \mu \mathrm{g} \mathrm{mL} \mathrm{m}^{-1}$ para fipronil, dessulfinil, sulfeto e sulfona e de 0,$5 ; 1,0 ; 2,0 ; 5,0$ e $10,0 \mu \mathrm{g} \mathrm{mL}^{-1}$ para atrazina, a equação da reta e os coeficientes de determinação $\left(R^{2}\right)$ foram obtidos para cada agrotóxico estudado. Os limites de deteç̧ão (LD) e limites de quantificação (LQ) do método foram determinados pelo procedimento de THIER \& ZEUMER (1987), considerando o menor nível de fortificação. A exatidão do método foi avaliada mediante estudos de recuperação das amostras de solo fortificadas e não fortificadas (testemunha) $(\mathrm{n}=3)$. A precisão foi obtida pelo coeficiente de variação do ensaio de recuperação.

Amostras de solo na profundidade de 0$10 \mathrm{~cm}$ foram coletadas nos campos experimentais da Embrapa Agropecuária Oeste em Dourados-

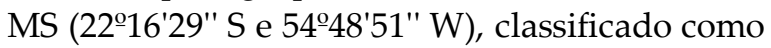
Latossolo Vermelho Distroférrico típico de textura argilosa (LVdf) e em Ponta Porã-MS (223'ㅇ' $06^{\prime \prime}$ S e 533'ㄱ' W), classificado como Latossolo Vermelho Distrófico típico de textura média (LVd) (SANTOS et al., 2006). As amostras foram secas à sombra, destorroadas, peneiradas em malha de $2 \mathrm{~mm}$ e homogeneizadas. Os atributos físico-químicos de ambos os solos estão apresentadas na Tabela 1. 
Tabela 1: Atributos químicos e físicos do Latossolo Vermelho Distroférrico típico de textura argilosa LVdf e do Latossolo Vermelho Distrófico típico de textura média - LVd.

\begin{tabular}{ccccccc}
\hline Solo & $\begin{array}{c}\text { Prof. } \\
(\mathbf{c m})\end{array}$ & $\begin{array}{c}\text { M.O. } \\
\left(\mathbf{g ~ k g ~}^{-1}\right)\end{array}$ & $\begin{array}{c}\text { Areia } \\
\left(\mathbf{g ~ k g ~}^{-1}\right)\end{array}$ & $\begin{array}{c}\text { Argila } \\
\left(\mathbf{g ~ k g ~}^{-1}\right)\end{array}$ & $\begin{array}{c}\mathbf{p H} \\
\left(\mathrm{CaCl}_{2}\right)\end{array}$ & $\begin{array}{c}\mathbf{Q} \\
\left(\mathbf{g ~ c m ~}^{-3}\right)\end{array}$ \\
\hline LVdf & $0-10$ & 33,4 & 245 & 630 & 4,8 & 1,21 \\
LVd & $0-10$ & 17,5 & 743 & 213 & 4,9 & 1,46 \\
\hline
\end{tabular}

M.O.: Matéria orgânica; @: densidade do solo

O procedimento empregado para validação do método consistiu na fortificação das amostras com adição das soluções dos padrões dos agrotóxicos em $10 \mathrm{~g}$ de solo seco. Para extração, adicionou-se $40 \mathrm{~mL}$ de metanol P.A ACS ISO (Vetec Brasil) e posteriormente agitouse durante 1 hora, deixando-se decantar por 1 hora. Alíquotas de $10 \mathrm{~mL}$ foram retiradas e colocadas em ultracentrífuga a $4000 \mathrm{rpm}$ durante 20 minutos, sendo filtradas em seguida utilizando filtro de seringa de celulose regenerada $0,45 \mu \mathrm{m}$. Todas as amostras foram armazenadas a $-20{ }^{\circ} \mathrm{C}$ até o momento da injeção por CLAE. Os níveis de recuperação avaliados foram de 0,$5 ; 2$ e $10 \mu \mathrm{g} \mathrm{mL}^{-1}$ para atrazina e $1 ; 5$ e $20 \mu \mathrm{g} \mathrm{mL} \mathrm{m}^{-1}$ para fipronil e seus produtos de degradação. Os analitos foram determinados por CLAE/DAD e a quantificação realizada pela comparação das áreas dos picos nas amostras com a curva de calibração obtida com as injeções dos padrões analíticos.

\section{Resultados e discussão}

A identificação do fipronil e seus produtos de degradação (dessulfinil, sulfeto, sulfona) e atrazina foi realizada empregando o detector de arranjo de diodos com base nos espectros de absorção molecular e no comprimento de onda de máxima absorção $\left(\lambda_{\text {máx }}\right)$, sendo de $210 \mathrm{~nm}$ para fipronil, dessulfinil, sulfeto e sulfona e de $220 \mathrm{~nm}$ para a atrazina (Figura 3). O comprimento de onda escolhido para execução das análises foi o de $220 \mathrm{~nm}$, pois em $210 \mathrm{~nm}$ aparecem alguns interferentes oriundos dos próprios solventes utilizados como, por exemplo, metanol e acetonitrila.

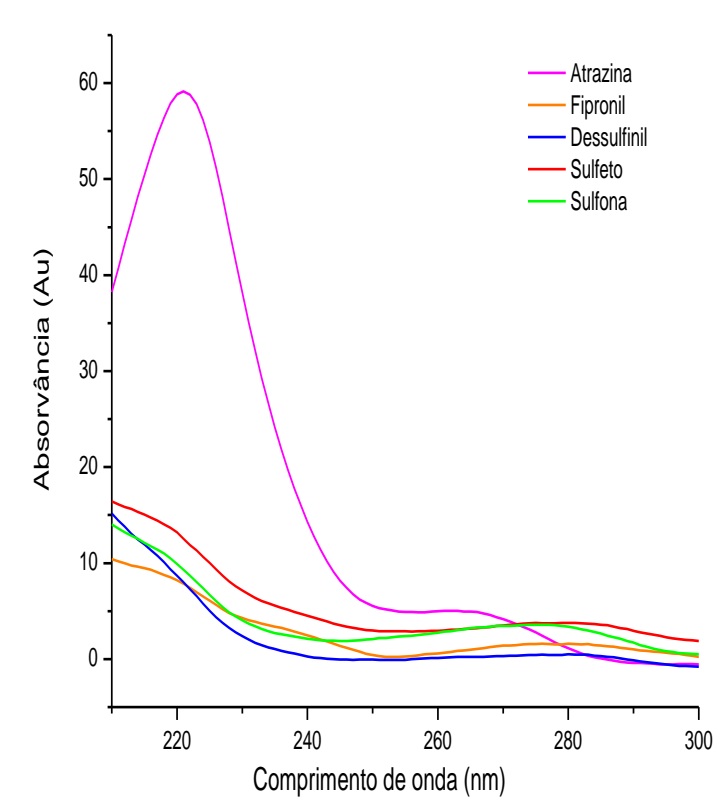

Figura 3. Espectro de absorção da análise por CLAE-DAD da solução de fipronil, dessulfinil, sulfeto, sulfona e atrazina na concentração de 5,0 $\mu \mathrm{g} \mathrm{mL} \mathrm{m}^{-1} \mathrm{em}$ metanol.

Não se observou a presença de interferentes nos tempos de retenção dos agrotóxicos estudados, nos cromatogramas das amostras testemunha (branco) em ambos os solos (Figura 4). Isso mostra a seletividade do método desenvolvido para determinação e quantificação dos agrotóxicos avaliados. Os picos da atrazina e do fipronil e seus produtos de degradação (dessulfinil, sulfeto e sulfona), mostrados na Figura 4, apresentaram boa resolução 


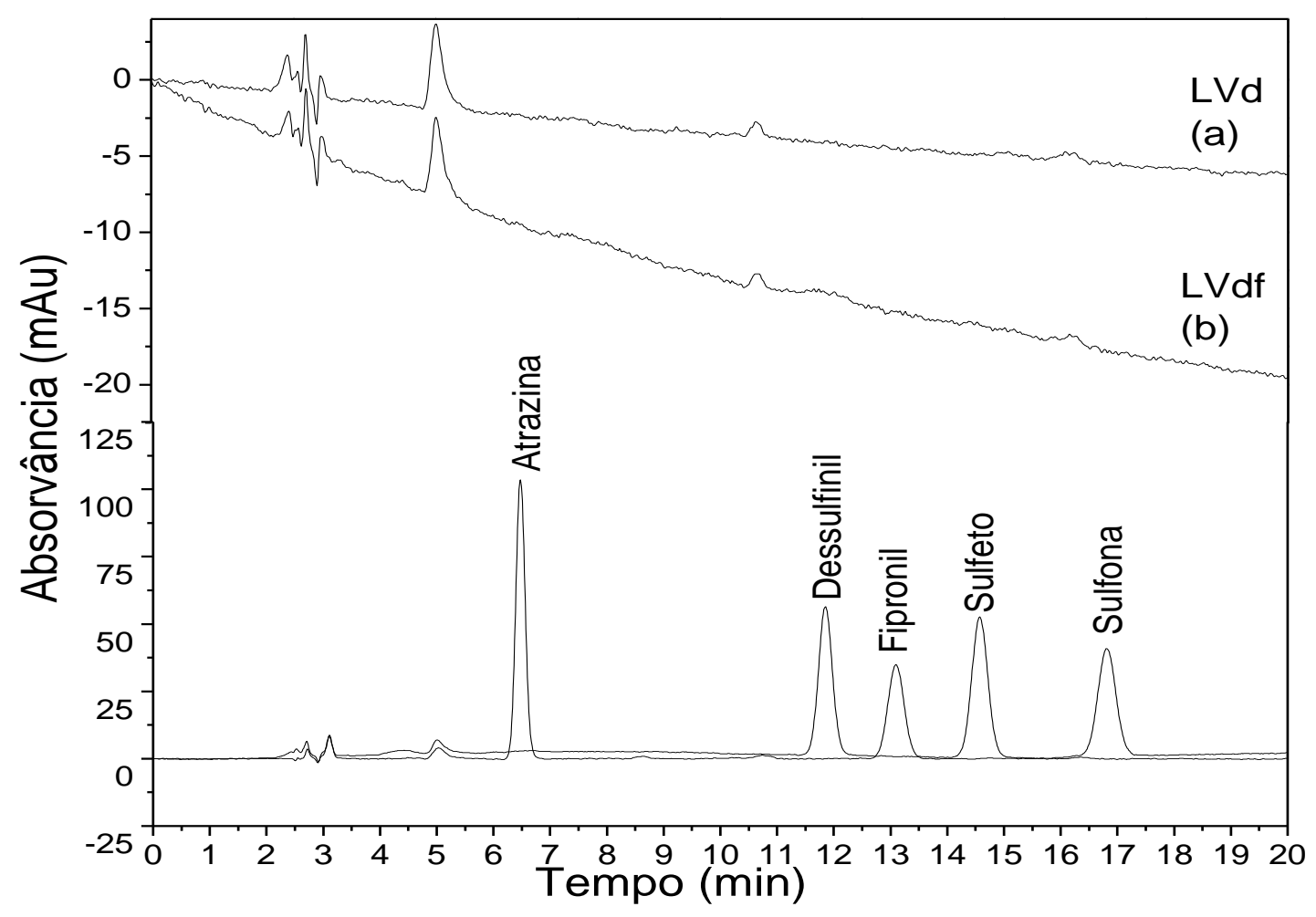

Figura 4. Cromatogramas das amostras testemunha (branco): Latossolo Vermelho Distrófico típico de textura média - LVd (a) e Latossolo Vermelho Distroférrico típico de textura argilosa - LVdf (b), padrões analíticos de atrazina $(10 \mu \mathrm{g} \mathrm{mL}-1)$ e fipronil e seus produtos de degradação $\left(20 \mu \mathrm{g} \mathrm{mL}^{-1}\right)$.

O método do padrão externo foi utilizado para a construção das curvas analítica, obtendo-se coeficiente de determinação $\left(R^{2}\right)$ superiores a 0,9997 (Tabela 2), obtidos nas faixas lineares de 0,5 a $10 \mu \mathrm{gL}^{-1}$ para atrazina, e de 1 a $20 \mu \mathrm{g} \mathrm{mL} \mathrm{m}^{-1}$ para fipronil e seus produtos de degradação (desulfinil, sulfeto e sulfona), o que mostra um bom resultado baseado nos valores de referência utilizados, o INMETRO (2010) recomenda valores de $\mathrm{R}^{2}$ maiores que 0,90 e a ANVISA (2003) igual a 0,99. A partir da regressão linear obteve-se a equação da reta: $y=a$ $+\mathrm{bx}$, onde (y) corresponde a área do pico, (a) coeficiente linear, (b) coeficiente angular e (x) concentração do analito (Tabela 2).

Os limites de detecção foram de $0,66 \mu \mathrm{g}$ $\mathrm{g}^{-1}$ para atrazina e de $1,32 \mu \mathrm{g} \mathrm{g}{ }^{-1}$ para fipronil e seus produtos de degradação (Tabela 2). Os limites de quantificação apresentaram valores de $4 \mu \mathrm{g} \mathrm{g}^{-1}$ para o fipronil e seus produtos de degradação e de $2 \mu \mathrm{g} \mathrm{g}^{-1}$ para atrazina (Tabela 2), com coeficientes de variação menores que $5 \%$ para todas as concentrações. A integração foi feita manualmente pico a pico, considerando a área do pico para quantificação (DYSON, 1998).

O método analítico torna-se eficiente em análise de resíduos de agrotóxicos, quando se encontram percentuais de recuperações na faixa de 70 a $120 \%$, e é considerado preciso quando o coeficiente de variação para as amostras é menor que $20 \%$ (RIBANI et al., 2004). Assim, pode-se concluir que o método testado para extração dos agrotóxicos no solo apresentou uma boa recuperação, com valores entre 79 e $93 \%$ para atrazina, 96 e $106 \%$ para o dessulfinil, 72 e $99 \%$ para o fipronil, 86 e $110 \%$ para o sulfeto e 79 a $110 \%$ para sulfona (Tabela 3). Os valores de coeficiente de variação foram menores que $13 \%$ (Tabela 3). A exatidão $(72-110 \%)$ e a precisão $(<13 \%)$ do método foram consideradas satisfatórias para a recuperação dos agrotóxicos estudados nos três níveis de fortificação analisados. 
Tabela 2. Parâmetros analíticos de validação do método.

\begin{tabular}{|c|c|c|c|c|c|c|}
\hline Agrotóxicos & $\begin{array}{c}\text { TR } \\
(\min )\end{array}$ & $\begin{array}{l}\text { Faixa linear } \\
\left(\mu \mathrm{g} \mathrm{mL} \mathrm{L}^{-1}\right)\end{array}$ & $\mathbf{R}^{2}$ & $\begin{array}{c}\text { LQ } \\
\left(\mu \mathrm{g} \mathrm{g}^{-1}\right)\end{array}$ & $\begin{array}{c}\text { LQ } \\
\left(\mu \mathrm{g} \mathrm{g}^{-1}\right)\end{array}$ & $\begin{array}{l}\text { Inclinação } \\
y=a+b x\end{array}$ \\
\hline Atrazina & 6,43 & $0,5-10,0$ & 0,9998 & 0,66 & 2,0 & $y=5,83493 \cdot 10^{-3}+2,47839 x$ \\
\hline Dessulfinil & 11,81 & $1,0-20,0$ & 0,9997 & 1,32 & 4,0 & $y=-0,14689+0,80907 x$ \\
\hline Fipronil & 12,88 & $1,0-20,0$ & 0,9999 & 1,32 & 4,0 & $y=0,02609+0,72880 x$ \\
\hline Sulfeto & 14,55 & $1,0-20,0$ & 0,9997 & 1,32 & 4,0 & $y=0,07760+0,86472 x$ \\
\hline Sulfona & 16,55 & $1,0-20,0$ & 0,9999 & 1,32 & 4,0 & $y=-0,04510+0,77028 x$ \\
\hline
\end{tabular}

TR=Tempo de retenção; $\mathrm{LD}=$ limite de detecção do método; $\mathrm{LQ}$ = limite de quantificação do método 
Tabela 3. Percentuais de recuperação e seus coeficientes de variação em três níveis de fortificação para atrazina, fipronil e seus produtos de degradação em dois solos de Mato Grosso do Sul (Latossolo Vermelho distroférrico típico de textura argilosa - LVdf e Latossolo Vermelho Distrófico típico de textura média - LVd.

\begin{tabular}{|c|c|c|c|c|c|}
\hline \multirow{3}{*}{ Agrotóxicos } & \multirow{3}{*}{$\begin{array}{c}\text { Níveis } \\
\text { de fortificação } \\
\left(\mu \mathrm{g} \mathrm{mL}^{-1}\right)\end{array}$} & \multicolumn{4}{|c|}{ Tipos de solo } \\
\hline & & \multicolumn{2}{|c|}{ LVdf } & \multicolumn{2}{|c|}{ LVd } \\
\hline & & $\begin{array}{c}\text { Recuperação } \\
(\%)\end{array}$ & CV $(\%)$ & $\begin{array}{c}\text { Recuperação } \\
(\%)\end{array}$ & CV (\%) \\
\hline \multirow{3}{*}{ Atrazina } & 0,5 & $79 \pm 2,6$ & 5,7 & $87 \pm 2,0$ & 4,1 \\
\hline & 2,0 & $87 \pm 4,1$ & 8,1 & $93 \pm 3,4$ & 6,4 \\
\hline & 10,0 & $81 \pm 1,4$ & 3,1 & $77 \pm 2,3$ & 5,2 \\
\hline \multirow{3}{*}{ Dessulfinil } & 1,0 & $106 \pm 1,2$ & 1,9 & $96 \pm 5,3$ & 9,5 \\
\hline & 5,0 & $102 \pm 2,3$ & 3,9 & $103 \pm 0,8$ & 1,4 \\
\hline & 20,0 & $96 \pm 0,9$ & 1,6 & $97 \pm 0,7$ & 1,3 \\
\hline \multirow{3}{*}{ Fipronil } & 1,0 & $84 \pm 4,9$ & 10,0 & $72 \pm 1,7$ & 4,0 \\
\hline & 5,0 & $99 \pm 4,5$ & 7,9 & $92 \pm 7,0$ & 13 \\
\hline & 20,0 & $81 \pm 2,0$ & 2,0 & $79 \pm 1,4$ & 3,2 \\
\hline \multirow{3}{*}{ Sulfeto } & 1,0 & $96 \pm 0,2$ & 0,3 & $86 \pm 5,2$ & 10,5 \\
\hline & 5,0 & $110 \pm 2,2$ & 3,6 & $105 \pm 0$ & 0 \\
\hline & 20,0 & $96 \pm 0,9$ & 1,6 & $97 \pm 0,7$ & 1,3 \\
\hline \multirow{3}{*}{ Sulfona } & 1,0 & $79 \pm 1,4$ & 3,2 & $110 \pm 3,1$ & 4,9 \\
\hline & 5,0 & $99 \pm 1,6$ & 2,8 & $87 \pm 5,9$ & 11,8 \\
\hline & 20,0 & $96 \pm 1,1$ & 1,9 & $99 \pm 0,6$ & 1,1 \\
\hline
\end{tabular}

Média \pm erro padrão, $(\mathrm{n}=3)$

A exatidão também pode ser observada na comparação dos cromatogramas dos padrões analíticos e das amostras fortificadas durante o teste de recuperação dos diferentes agrotóxicos avaliados nas concentrações de $20 \mu \mathrm{g} \mathrm{mL}^{-1}$ para o fipronil, dessulfinil, sulfeto e sulfona e de $10 \mu \mathrm{g}$ $\mathrm{mL}^{-1}$ para atrazina (Figura 5).

A vantagem deste método validado por CLAE é que permite a detecção simultânea dos agrotóxicos fipronil e seus produtos de degradação, bem como da atrazina, e não precisa de purificação das amostras.

Nos métodos desenvolvidos para Cromatografia gasosa com detector de captura de elétrons (GC-ECD) a amostra precisa passar por uma longa etapa (aproximadamente 7 dias) de purificação (CHOPRA et al., 2011; RAMÍREZ et al., 2012; SAINI et al., 2014). 


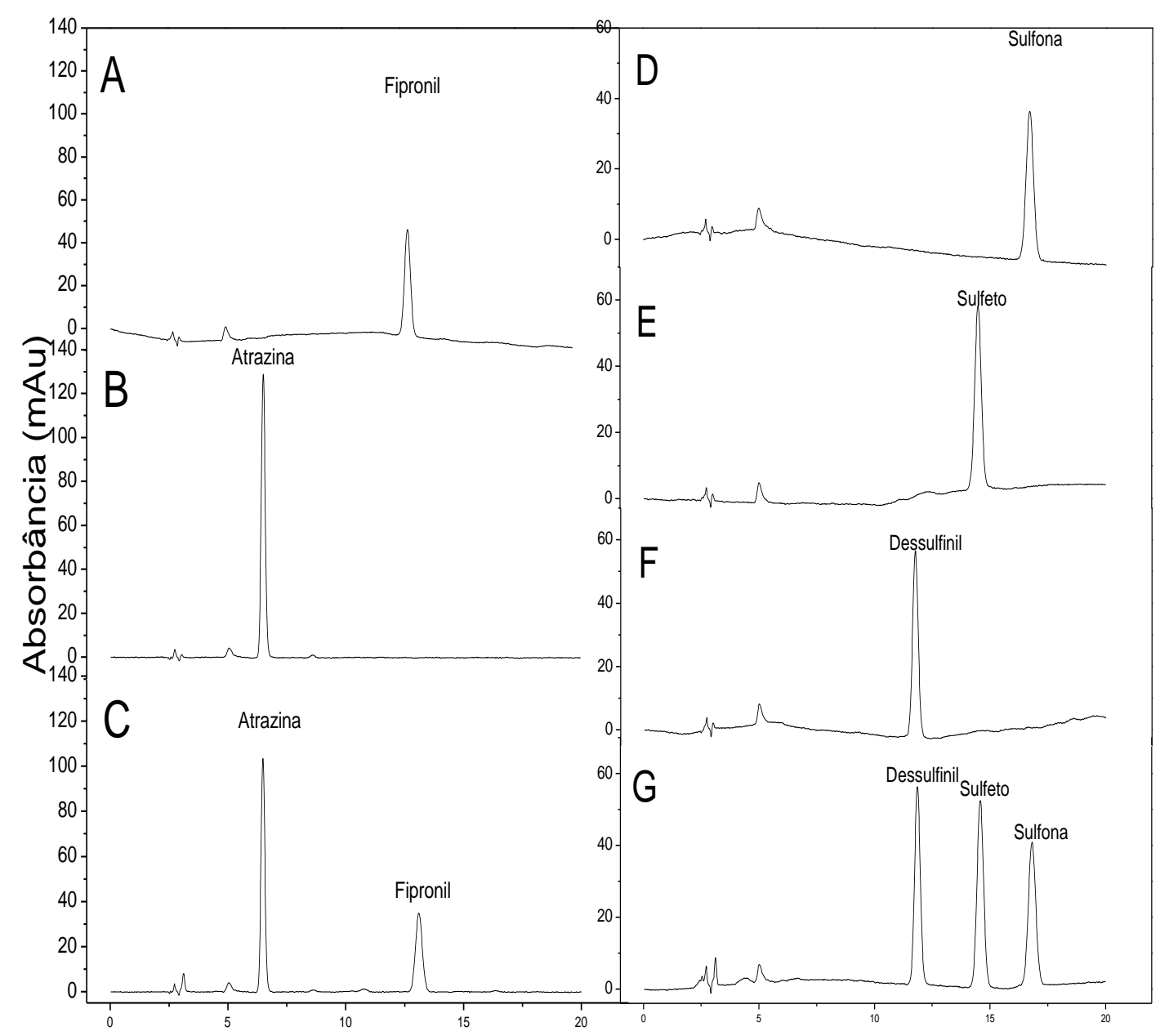

Figura 5. Cromatogramas dos padrões analíticos de fipronil (A), atrazina (B), sulfona (D), sulfeto (E), dessulfinil (F) e amostras fortificadas com os padrões de fipronil e atrazina (C) e desulfinil, sulfeto e sulfona $(G)$.

O método validado por CLAE-DAD utiliza uma pequena quantidade de solvente para extração, produzindo um método mais sustentável, sendo esta uma vantagem comparada aos métodos desenvolvidos por HADJMOHAMMADI et al. (2006); MASUTTI \& MERMUT, (2007); MOHAPATRA \& AHUJA, (2010); MANDAL \& SINGH, 2013, que utilizam grande quantidade de solvente. $\mathrm{O}$ método desenvolvido diminui o tempo de análise em relação a outros descritos na literatura, principalmente em relação ao preparo e purificação das amostras.

\section{Conclusão}

O método proposto para extração e quantificação simultânea de resíduos de fipronil, dessulfinil, sulfeto, sulfona e atrazina em amostras de solo mostrou-se satisfatório, visto que é de fácil e rápida execução. Assim, este método pode ser utilizado em laboratórios com objetivo de identificar e quantificar resíduos de atrazina, fipronil e seus produtos de degradação em amostras de solo, sem necessidade de purificação das amostras.

\section{Agradecimentos}

A Coordenação de Aperfeiçoamento de Pessoal de Nível Superior (CAPES) pelo incentivo financeiro e pela bolsa de mestrado, Conselho Nacional de Desenvolvimento Científico e Tecnológico (CNPq) pela bolsa de PQ de C. A. L. Cardoso e a Embrapa Agropecuária Oeste pelo apoio técnico no projeto. 


\section{Referências}

AGROFIT: Sistema de Agrotóxicos

Fitossanitários. Brasília: Ministério da Agricultura, Pecuária e Abastecimento, 2003.

ANDREU, V.; PICÓ Y. Determination of pesticides and their degradation products in soil: Critical review and comparison of methods. Trends in Analytical Chemical, v. 23, p. $772-$ 789, 2004.

ANVISA: Agência Nacional de Vigilância Sanitária. Resolução RE nº 899, de 29/05/2003

ARIAS-ESTEVEZ, M.; LOPEZ-PERIAGO, E.; MARTINEZ-CARBALLO, E.; SIMALGANDARA, J.; MEJUTO, J. C.; GARCIARIO, L. The mobility and degradation of pesticides in soils and the pollution of groundwater resources. Agriculture, Ecosystems and Environment, v.123, p. 247-260, 2008.

BASF:

Disponível

em:

http://www.agro.basf.com.br/agr/ms/apbrazil/pt _BR/content/APBrazil/solutions/insecticides/inse cticides_product/Regente_800_WG. Acesso em 27 mai. 2015.

BARCELÓ, D.; HENNION, M. C. Trace determination of pesticides and their degradation products en water. 3 ed. Amsterdam: Elsevier, 1997, 542 p.

BOBÉ, A.; COOPER, J. F.; COSTE, C. M.; MULLER, M. A. Behavior os fipronil em soil under sahelian plain field conditions. Pesticides Science, v. 52, p. 275-281, 1998.

CHOPRA, I.; CHAUHAM, R.; KUMARI, B.; DAHIYA, K. K. Fate of Fipronil in Cotton and Soil Under Tropical Climatic Conditions. Bulletin of Environmental Contamination and Toxicology, v. 86, p. 242-245, 2011.

HADJMOHAMMADI, M. R.; NIKOU, S. M.; KAMEL, K. Determination of Fipronil Residue in Soil and Water in the Rice Fields in North of Iran by RP-HPLC Method. Acta Chimica Slovenica, v. 53, p. 517-520, 2006.
HAYES, T. B.; ANDERSON, L. L.; BEASLEY, V. R.; SOLLA, S. R.; IGUCHI, T.; INGRAHAM, $\mathrm{H}$.; et al. Demasculinization and feminization of male gonads by atrazine: Consistent effects across vertebrate classes. Journal of Steroid Biochemistry \& Molecular Biology, v. 127 p. 6473, 2011.

INMETRO: Instituto Nacional de Metrologia, Normalização e Qualidade Industrial. Orientações sobre Validação de Métodos de Ensaios Químicos, DOQ-CGCRE-008, 2010. Disponível em: http://www.inmetro.gov.br/Sidoq/Arquivos/CG CRE/DOQ/DOQ-CGCRE-8_03.pdf. Acesso em 10 de jun. de 2015.

MANDAL, K.; SINGH, B. Persistence of fipronil and its metabolites in sandy loam and clay loam soils under laboratory conditions. Chemosphere, v. 91, p1596-1603, 2013.

MAPA Ministério da Agricultura, Pecuária e Abastecimento. Disponível em: http :// extranet. agricultura.gov.br/agrofit_cons/principal_agrofit _ cons. Acesso em mar 2015.

MARTINAZZO, R.; DICK, D. P.; HIRSCHI, M. M.; LEITE, S. B.; PERELBA, M. C. R. Sorção da atrazina e de mesotriona em latossolo e estimativa de potencial de contaminação. Química Nova, v. 34, n. 8, p. 1378-1384, 2011.

MASUTTI, C. S. M.; MERMUT, A. R. Degradation of fipronil under laboratory conditions in a tropical soil from sirinhaém pernambuco, Brazil. Journal of Environmental Science and Health Part B, v. 42, p. 33-43, 2007.

MOHAPATRA, S.; AHUJA, A. K. Behaviour of fipronil in soil under different moisture levels. Indian Journal of Agricultural Sciences, v. $80 \mathrm{n}$. 7, p. 658-61, 2010.

MONTGOMERY, J. H. Agrochemicals, desk reference. 2 ed. Boca Raton, New York, Lews Publichers, 1997, 656p.

Pesticide Action Network-UK (PAN). (2000). Active ingredient Fact Sheet: Fipronil, Pesticides News London, v. 48, p. 5-30. 
PERES, T. B.; PAPINI, S.; MARCHETTI, M.; NAKAGAWA, L. E.; MARCONDES, M. A.; ANDRÉA, M. M. ; LUCHINI, L. C. Métodos de extração de agrotóxicos de diversas matrizes, Arquivos do Instituto Biológico, v. 69, n. 4, p. 87-94, 2002.

RAMÍREZ, R. F.; ESQUIVEL, L. E. B.; MARTÍNEZ, F. D. B.; ACOSTA, I. L.; PÉREZ, M. D. O. Development and Validation of an Analytical Method to Determine Fipronil and its Degradation Products in Soil Samples. Bulletin of Environmental Contamination and Toxicology, v. 89, p. 744-750, 2012.

RIBANI, M.; BOTTOLI, C. B. G.; COLLINS, C. H.; JARDIM, I. C. S. F.; MELO, L. F. C. Validação em métodos cromatográficos e eletroforéticos. Química Nova, v. 27, p. 771-780, 2004.

SANTOS, H. G.; JACOMINE, P. K. T.; ANJOS, L. H. C.; OLIVEIRA, V. A.; OLIVEIRA, V. B.; COELHO, M. R.; et al. Sistema Brasileiro de Classificação de Solos. 2. ed. Rio de Janeiro: Solos, 2006. 306 p.

SAINI, S.; RANI, M.; KUMARI, B. Persistence of fipronil and its metabolites in soil under field conditions. Environmental Monitoring and Assessment, v. 186, n. 1, p. 69-75, 2014.

DYSON, N. A. Chromatographic integration methods. 2. ed. Loughborough, UK. The Royal Society of Chemistry, 1998. 224p.

SPADOTTO , C. A.; GOMES, M. A. F.; LUCHINI, L. C.; ANDÉA, M. M. Monitoramento de risco ambiental de agrotóxicos: princípios e recomendações. Jaguariúna: Embrapa Meio Ambiente, 2004. 29 p. (Documento, 42).

THIER, H.P.; ZEUMER, H. Manual of pesticide residue analysis, New York: VHC, 1987, 218p.

U.S. EPA, 1996. New pesticide fact sheet. PB96-181516. EPA 737-F-96-005. U.S. EPA Office of Prevention, Pesticides and Toxic Substances. 